\title{
Controle da malária no Brasil: 1965 a 2001
}

\author{
Carlos Catão Prates Loiola, ${ }^{1}$ C. J. Mangabeira da Silva ${ }^{2}$ \\ e Pedro Luiz Tauil ${ }^{3}$
}

RESUMO O objetivo deste trabalho foi revisar as iniciativas para controle da malária no Brasil, desde a Campanha de Erradicação da Malária, de 1965, baseada na ação do diclorodifeniltricloroetano e no uso de drogas antimaláricas, até o lançamento, em 2000, do Plano de Intensificação das Ações de Controle da Malária na Amazônia Legal, em resposta à convocação da Organização Mundial da Saúde para fazer recuar a malária (Roll Back Malaria). São discutidas iniciativas como a estratificação epidemiológica, Operação Impacto, Projeto de Controle da Malária na Bacia Amazônica e Programa de Controle Integrado da Malária. Embora tenha havido avanços antes do Plano de Intensificação, não houve sustentabilidade das ações. Entre 1998 e 1999, houve inclusive um aumento de $34 \%$ no número de casos de malária nos estados amazônicos. O Plano de Intensificação das Ações de Controle da Malária na Amazônia Legal estabeleceu a meta de reduzir em $50 \%$ o número de casos de malária até o final de 2001 e em 50\% a mortalidade por malária até o final de 2002, em comparação com o ano de 1999. Os dados de 2001 revelam uma redução de $39 \%$ no número de casos de malária em todos os estados amazônicos. A menor redução ocorreu no Amapá (15\%), onde o Plano só foi implantado no $2^{\circ}$ semestre de 2001. Em relação à incidência por espécies, a redução global de casos por P. falciparum foi de 35\%, e por P. vivax, de $41 \%$. Para explicar tal redução, a única variável independente reconhecida foi a implantação do Plano de Intensificação. Embora ainda precoces, os resultados sugerem um considerável ganho, onde uma forte mobilização política dos três níveis de governo - federal, estadual e municipal - foi decisiva.

Palavras chave Malária, prevenção \& controle, descentralização, programas de governo, ecossistema amazônico.

A história dos esforços para controlar a malária no Brasil remonta, provavelmente, ao final do século XIX e início do século XX. Contudo, o presente trabalho procura-se situar dentro de um período que se inicia em 1965, com a criação da Campanha de Erradicação

\footnotetext{
1 Representação da Organização Pan-Americana da Saúde no Brasil. Correspondência e pedidos de separatas devem ser encaminhados a este autor no seguinte endereço: Setor de Embaixadas Norte, Lote 19, caixa postal 08729, CEP 70800-400, Brasília, DF, Brasil. E-mail: catao@bra.ops-oms.org

2 Ministério da Saúde, Fundação Nacional de Saúde.

3 Universidade de Brasília, Faculdade de Medicina.
}

da Malária, até o momento, em 2000, quando se deu o lançamento, pelo Ministério da Saúde/Fundação Nacional de Saúde (FUNASA), de um novo esforço para controlar a malária - o Plano de Intensificação das Ações de Controle da Malária na Amazônia Legal (PIACM).

\section{A CAMPANHA DE ERRADICAÇÃO DA MALÁRIA}

É incontestável o fato de que a estratégia de erradicação da malária preconizada pela Organização Mundial da
Saúde (OMS) e adotada pelo Brasil a partir de 1965, baseada na ação intradomiciliar do diclorodifeniltricloroetano (DDT) contra os anofelinos transmissores e no uso de drogas antimaláricas para esgotamento das fontes de infecção (seres humanos parasitados pelos plasmódios), foi capaz de eliminar a malária de extensas áreas do território brasileiro (regiões Nordeste, Sudeste, Centro-Oeste e Sul) onde uma parcela significativa da nossa população vivia sob o risco de contrair a malária. Cerca de 40 milhões de brasileiros (1) estiveram, nessas áreas, expostos a algum risco de contrair esta 
doença até 1979, quando tais áreas foram consideradas livres da transmissão autóctone de malária. É importante ressaltar que, além da presença do Anopheles $(N)$ darlingi, principal vetor no país, havia, em todas as regiões citadas, uma predominância muito maior de outras espécies transmissoras de malária, tais como Anopheles (N) albitarsis, Anopheles (N) aquasalis, Anopheles (K) cruzii e Anopheles (K) bellator.

Para erradicar a malária, o governo brasileiro criou, em 1965, através de uma lei (4709, de 6 de setembro de 1965), a Campanha de Erradicação da Malária (CEM). A CEM tinha autonomia administrativa e financeira, quadro de pessoal e orçamento próprios, era organizada dentro dos princípios rígidos da disciplina e hierarquia e tinha capacidade técnica e operacional suficientes para executar, verticalmente, suas ações de cobertura integral das medidas de controle em todas as áreas maláricas do país.

Durante o período de vigência da CEM, de acordo com os dados epidemiológicos registrados, houve um significativo e importante impacto na transmissão da malária (tabela 1). Conforme Marques e Gutierrez (2), no período de 1967 a 1969 elevou-se o número dos exames realizados $(1,7$ milhão como média anual) ao mesmo tempo em que houve uma diminuição no número dos exames positivos $(74,6$ mil, em média, ao ano).

\section{O CONTROLE DA MALÁRIA NA AMAZÔNIA BRASILEIRA}

Durante muito tempo, o Brasil insistiu em aplicar na Amazônia a mesma estratégia da CEM, baseada na aplicação intradomiciliar do DDT e no uso de drogas antimaláricas. Contudo, as características da região, onde predominavam habitações precárias, sem superfícies que permitissem uma aplicação adequada e correta do DDT, colocava a estratégia sob o risco do insucesso, fato que logo se confirmou. Não era possível sustentar uma proposta baseada na aplicação intradomiciliar de um inseticida de ação residual onde não se podia aplicá-lo por ausência de superfícies borrifáveis. Não bastasse esse fato, observava-se que a transmissão extradomiciliar na região tinha um peso significativo, que comprometia a efetividade do uso intradomiciliar. Somado a isso, começaram a surgir os primeiros sinais de resistência do P. falciparum à droga cloroquina, o que tornava ainda mais inatingível a perspectiva da erradicação.

Entre os fatores que podem ter contribuído para o insucesso da CEM na Amazônia estão: 1) presença de floresta tropical úmida, favorecendo $\mathrm{o}$ desenvolvimento e proliferação dos vetores da doença; 2) presença de grupos humanos especialmente expostos ao contato com os vetores: garimpeiros, madeireiros, agricultores em assentamentos de colonização; 3) alta incidência de $P$. falciparum resistente aos antimaláricos seguros para uso no campo; e 4) ausência de infra-estrutura social e de serviços permanentes de saúde, na grande maioria dos municípios. Tudo isso, com certeza, contribuiu para reduzir a efetividade das medidas.

Na XXII Assembléia Mundial da Saúde, em 1969, recomendou-se a classificação das áreas maláricas em áreas de erradicação a curto e longo prazo. Ainda insistia-se que a erradicação era possível, mesmo que a longo prazo. Verificou-se, mais tarde, que essa proposta era insustentável, e os países passaram a adotar a estratégia do controle em virtude da existência de fatores epidemiológicos, entomológicos, econômicos e sociais predominantes que impediam a erradicação da malária, mesmo que a longo prazo.

Enquanto isso, no Brasil, fenômenos relacionados ao processo de desenvolvimento da Amazônia contribuíam para um considerável incremento da malária. A política desenvolvimentista e de ocupação daquela região na década de 1980 levou ao estabelecimento de um fluxo migratório imenso e intenso, na grande maioria de pessoas das regiões Nordeste, Centro-Oeste e Sul do país, sem nenhuma imunidade adquirida e, portanto, alvos mais que perfeitos para a malária.

Diante de um quadro cada vez mais grave de aumento da incidência da ma- lária, o poder público se mostrava incapaz de agir contra essa situação, não só em razão de fatores de natureza epidemiológica e social mas, e sobretudo, porque já havia perdido grande parte da sua capacidade operacional de ação, pois já não contava mais com a CEM. Naquele momento, a instituição responsável pelo controle da malária no país era a Superintendência de Campanhas de Saúde Pública (SUCAM), órgão criado em 1970 e que, apesar de seus grandes e reconhecidos méritos, não tinha a mesma capacidade e a mesma estrutura da antiga CEM, mesmo porque a SUCAM não se dedicava exclusivamente ao controle da malária.

Diante disso, várias iniciativas foram adotadas na tentativa de minimizar o problema e alcançar um controle sustentável da transmissão da malária na região Norte do país. Dentre elas, destacam-se algumas, julgadas mais importantes, que serão descritas a seguir.

\section{Estratificação epidemiológica}

Reconhecida a dificuldade de alcançar, de forma simultânea, todas as áreas da região amazônica com medidas de controle da malária, em virtude de fatores ambientais, epidemiológicos, sociais e econômicos já conhecidos, buscou-se uma estratégia mais adequada à cada realidade e ajustada à disponibilidade de recursos, tanto humanos quanto financeiros, para uma cobertura satisfatória com ações de controle da malária em áreas homogêneas e com características epidemiológicas semelhantes. A esta forma de atuar, ainda muito incipiente, deu-se o nome de estratificação epidemiológica, com base no levantamento e reconhecimento dos fatores de risco envolvidos no processo de produção da doença e, principalmente, na maior ou menor ocorrência de casos de malária.

No início dos anos 1980, tal estratégia começou a ser adotada pela SUCAM (3-5). Os estados do Pará e de Rondônia foram pioneiros na implantação da estratificação epidemiológica. Antes da estratificação, as operações de campo 
TABELA 1. Número de lâminas examinadas e positivas para malária, Brasil, 1965 a 1970

\begin{tabular}{|c|c|c|c|c|c|c|c|c|}
\hline \multirow[b]{2}{*}{ Ano } & \multicolumn{3}{|c|}{ Amostras de sangue } & \multicolumn{5}{|c|}{ Espécies parasitárias } \\
\hline & Examinadas & Positivas & ILPa & Falciparum & $\%^{\mathrm{b}}$ & Vivax & $\mathrm{F}+\mathrm{V}^{\mathrm{c}}$ & $M^{d}$ \\
\hline 1965 & 1665997 & 103516 & 6,2 & 45575 & 44 & 57100 & 727 & 114 \\
\hline 1966 & 1677572 & 106890 & 6,4 & 56764 & 53 & 49257 & 622 & 247 \\
\hline 1967 & 1709930 & 94924 & 5,6 & 51488 & 54 & 42783 & 435 & 218 \\
\hline 1968 & 1732157 & 76419 & 4,4 & 40894 & 54 & 34913 & 384 & 228 \\
\hline 1969 & 1854857 & 52400 & 2,8 & 27611 & 53 & 24265 & 376 & 148 \\
\hline 1970 & 2012450 & 52469 & 2,6 & 26248 & 50 & 25722 & 348 & 151 \\
\hline
\end{tabular}

Fonte: Gerência Técnica de Malária/Fundação Nacional de Saúde.

a Índice de lâminas positivas por 100 lâminas examinadas.

b Porcentagem da espécie falciparum em relação ao total de espécies identificadas.

c Plasmodium falciparum + Plasmodium vivax.

d Plasmodium malariae.

para controle de vetores e de tratamento eram direcionadas pelos resultados obtidos pelos exames realizados em laboratório (localidades identificadas como positivas pela origem dos casos). Com a estratificação, e reconhecendo-se a necessidade de otimizar a aplicação dos recursos, buscou-se, mediante uma análise epidemiológica mais apurada, a seleção de áreas de maior transmissão nos municípios, identificando-se as localidades geradoras e, com isso, aquelas com maior ou menor dispersão e difusão da malária, o que possibilitou estabelecer as prioridades I (áreas onde foram concentrados todos os recursos humanos, materiais e financeiros) e II (áreas onde foram aplicados os excedentes dos recursos aplicados nas áreas de prioridade I), para efeito operacional imediato.

De qualquer forma, a ação era contida pela considerável limitação de recursos. E, como outras iniciativas, esta também fracassou por falta de sustentabilidade, gerada, principalmente, pela insuficiência de apoio logístico de transporte, inseticida, medicamentos, equipamentos (microscópios e bombas aspersoras) e peças de reposição.

\section{Operação Impacto}

Formulada e operacionalizada no ano de 1986, com poucos meses de duração, a Operação Impacto caracterizou-se por uma imensa mobilização de recursos, tanto humanos quanto materiais e financeiros. Planejada para ser uma ação intensa e de curto prazo, dirigida a reduzir rapidamente os níveis de morbimortalidade nos estados de Mato Grosso, Pará e Rondônia, que concentravam mais de $80 \%$ da malária da região, teve como base estratégica os mesmos princípios da erradicação. Apenas um elemento novo foi introduzido nessa operação, que a diferenciava das estratégias anteriores: o "zoneamento", através do qual foram delimitadas áreas geográficas e populacionais para localização de forças-tarefas que garantiriam uma cobertura integral e simultânea com ações de tratamento humano em massa e controle de vetores. Previsto para ser implementado na etapa final da operação, como estratégia para dar sustentabilidade às ações executadas em três etapas, o zoneamento, na verdade, nunca chegou a ser concretizado. Foi nessa operação e nesse momento que o Brasil introduziu o uso da mefloquina para tratamento da malária por Plasmodium falciparum.

Segundo definição dada pela própria SUCAM, a Operação Impacto consistiu em uma mudança de estratégia operacional dos trabalhos de combate à malária e dividiu-se em duas partes: 1) aplicação, em três etapas, de medicamentos a pessoas residentes nos municípios de maior transmissão da malária; e 2) divisão dos municípios em pequenas áreas, cada uma a ser servida por um guarda da SUCAM fazendo borrifação das casas, coleta de lâmina dos febris, tratamento, eliminação dos criadouros de mosquitos e orientação à população $(3,6)$.

Quem deveria tomar a medicação? Na primeira etapa, todas as pessoas febris e todas aquelas residentes na casa; na segunda etapa, somente as pessoas febris; e na terceira etapa as pessoas que ainda estivessem com malária comprovada por exame de sangue. A justificativa para a utilização do tratamento coletivo empregado foi a possibilidade de reduzir, com essa medida, altas incidências de malária em áreas previamente selecionadas, onde os métodos tradicionais de ataque não vinham alcançando resultados satisfatórios no controle da doença.

Os medicamentos utilizados foram cloroquina, primaquina, pirimetamina, sulfadoxina e mefloquina. Esta última foi empregada na fase final do programa, para tratamento dos casos de malária produzidos por cepas de Plasmodium falciparum resistentes aos tratamentos anteriores e com diagnóstico parasitológico de confirmação realizado através do exame de sangue. Os esquemas terapêuticos empregados foram: para a pirimetamina $(25 \mathrm{mg})$ e a sulfadoxina $(500 \mathrm{mg})$, associação em um só comprimido, com administração em dose única (três comprimidos para maiores de 15 anos). Para a cloroquina foram utilizados comprimidos individualizados de $250 \mathrm{mg}$, contendo 150 $\mathrm{mg}$ de cloroquina/base, administrados 
durante 3 dias (quatro comprimidos no $1^{\circ}$ dia, três no $2^{\circ}$ dia e três no $3^{\circ}$ dia, associados a três comprimidos de primaquina de $15 \mathrm{mg}$ para maiores de 15 anos). A primaquina era administrada em comprimidos de $5 \mathrm{mg}$ para crianças e $15 \mathrm{mg}$ para adultos. A mefloquina era utilizada em comprimidos de 250 mg, administrados em dose única de quatro comprimidos associados a três comprimidos de primaquina de $15 \mathrm{mg}$ para maiores de 15 anos.

Sabe-se ainda que, para executar essa operação, foram deslocados para os estados do Pará, Mato Grosso e Rondônia servidores da SUCAM de várias regiões do país. Do Nordeste, do Centro-Oeste e do Sudeste foram mobilizados cerca de 2000 servidores, com apoio dos ministérios da Aeronáutica e do Exército para transporte às áreas onde deveriam atuar.

Essa operação, duramente criticada por muitos, não produziu, como se esperava, um impacto que justificasse um gasto tão elevado de recursos e a utilização de estratégias bastante questionadas, como por exemplo o uso indiscriminado da mefloquina para tratamento da malária em áreas hiperendêmicas.

Lamentavelmente, não se sabe exatamente quanto foi gasto com a operação e nem sequer se chegou a fazer uma avaliação da mesma, tanto do ponto de vista de resultados quanto do ponto de vista de custo-benefício. Os dados epidemiológicos disponíveis nos sistemas de registro da FUNASA mostram que não houve o impacto desejado (tabela 2).

\section{Projeto de controle da malária na bacia amazônica}

Diante de um quadro cada vez mais grave, e demandado pelo poder político regional, que via na presença da malária na Amazônia um fator impeditivo para o desenvolvimento daquela região, o Ministério da Saúde buscou alternativas de financiamento para a intensificação das ações de controle dessa endemia. Assim, encontrou receptividade junto ao Banco Mundial/Banco Internacional de Reconstrução e Desenvolvimento (BIRD) para financiamento do projeto de controle da malária na bacia amazônica (PCMAM) (7). Essa foi a origem do acordo de empréstimo BRA/3072, no valor de 99 milhões de dólares que, somados a outros US\$ 99 milhões de contrapartida nacional, totalizavam um montante de US\$ 198 milhões para serem aplicados em 5 anos — de 1989 até 1993.

Os objetivos desse projeto foram: 1) reduzir a ocorrência de casos de malária; 2) promover o desenvolvimento institucional da SUCAM e das secretarias estaduais de saúde; 3 ) fortalecer o controle da malária; e 4) dar atenção específica à saúde das comunidades indígenas.

Seguramente, o PCMAM colaborou muito para a estruturação dos serviços locais de saúde, principalmente quanto à capacidade de diagnóstico e tratamento e, também, para o avanço do processo de descentralização do controle das endemias. No curso desse projeto, mais precisamente no ano de
1991, ocorreu uma profunda transformação administrativa no âmbito do Ministério da Saúde, onde a SUCAM, na época o órgão responsável pela gestão e execução do PCMAM, foi incorporada à Fundação Serviços Especiais de Saúde Pública (FSESP) juntamente com áreas das antigas Secretaria Nacional de Programas Especiais de Saúde (SNEPS) e Secretaria Nacional de Ações Básicas de Saúde (SNABS), para se transformar na atual FUNASA.

Em razão, principalmente, das indefinições no âmbito do Ministério da Saúde e da FUNASA, e contando ainda com as enormes dificuldades administrativas vivenciadas naquele período, $\mathrm{o}$ PCMAM ficou praticamente paralisado de 1991 a 1993 e, assim, foi prorrogado por mais 3 anos, até junho de 1996. Além disso, houve um corte substancial no volume de recursos do empréstimo e, conseqüentemente na contrapartida nacional, o que significou uma redução da ordem de US\$ 40 milhões.

A partir de 1993, entretanto, o PCMAM retomou seu ritmo e alcançou uma execução de $100 \%$ dos recursos alocados, ou seja, foram aplicados e gastos todos os recursos destinados ao projeto. Além disso, conseguiu feitos importantíssimos, como a ampliação da rede de laboratórios para diagnóstico e tratamento da malária. Alcançou, também, um nível bastante satisfatório na capacitação de recursos humanos, com destaque para equipes técnicas - médicos e auxiliares - para manejo de pacientes com quadro grave de malária, tanto em nível ambulatorial como hospitalar.

TABELA 2. Lâminas examinadas e positivas para malária, Brasil, 1986 a 1988

\begin{tabular}{|c|c|c|c|c|c|c|c|c|}
\hline \multirow[b]{2}{*}{ Ano } & \multicolumn{3}{|c|}{ Amostras de sangue } & \multicolumn{5}{|c|}{ Espécies parasitárias } \\
\hline & Examinadas & Positivas & ILPa & Falciparum & $\%$ b & Vivax & $\mathrm{F}+\mathrm{V}^{\mathrm{c}}$ & $M^{d}$ \\
\hline 1986 & 3363962 & 443627 & 13,2 & 240664 & 54 & 199857 & 3097 & 9 \\
\hline 1987 & 3070540 & 508864 & 16,6 & 266148 & 52 & 238403 & 4310 & 3 \\
\hline 1988 & 3373283 & 559535 & 16,6 & 282786 & 51 & 271784 & 4964 & 1 \\
\hline
\end{tabular}

Fonte: Gerência Técnica de Malária/Fundação Nacional de Saúde.

a Índice de lâminas positivas por 100 lâminas examinadas.

b Porcentagem da espécie falciparum em relação ao total de espécies identificadas.

c Plasmodium falciparum + Plasmodium vivax.

d Plasmodium malariae. 
A conseqüência imediata desses feitos foi a importantíssima redução nas taxas de mortalidade por malária que, de 7 mortes por 1000 habitantes em 1988 caiu para 1,8 mortes por 1000 habitantes em 1995. Um estudo realizado pelo Banco Mundial (8) revelou que o PCMAM contribuiu para a redução da carga social produzida pela malária e também possibilitou retornou econômico altamente favorável ao investimento realizado. Esse estudo concluiu que, no período de 1988 a 1996, foram evitados cerca de 1,9 milhões de novos casos e 236 mil óbitos por malária. $\mathrm{O}$ mesmo estudo revelou ainda que houve um ganho de 8,8 milhões de anos de vida ajustados por incapacidade (AVAIS). Embora tenha-se investido bastante em treinamento de pessoal local, não se chegou a avaliar por que, apesar de tudo isso, o PCMAM não conseguiu estimular a criação de estruturas locais capazes de assumirem as ações de controle da malária.

Em quase todos os estados, a dependência direta do órgão federal ainda era muito evidente, tanto é que, ao término do PCMAM, com a retração nos investimentos por parte do governo federal, houve um recrudescimento da endemia com elevação da incidência (tabelas 3 e 4).

\section{Programa de controle integrado da malária}

Ainda durante a vigência do PCMAM, em 1992, ocorreu a Conferência Ministerial de Amsterdã, pro-

TABELA 3. Recursos financeiros aplicados no programa de controle da malária no Brasil, 1995 a 1998

\begin{tabular}{|c|c|c|c|c|}
\hline \multirow[b]{2}{*}{ Fonte } & \multicolumn{4}{|c|}{ Recursos (em US\$1000,00)a } \\
\hline & 1995 & 1996 & 1997 & 1998 \\
\hline BIRD & 42905 & $8821^{b}$ & - & - \\
\hline Tesouro federal & 36253 & 39155 & 33680 & 34941 \\
\hline Total & 79158 & 47976 & 33680 & 34941 \\
\hline
\end{tabular}

movida pela OMS, que estabeleceu uma nova estratégia para o controle da malária no mundo. Fortemente voltada para o indivíduo doente, com um pressuposto básico da necessidade de alcançar metas para consolidação de redes de serviços capazes de ofertar diagnóstico precoce e um tratamento correto e imediato, essa nova estratégia já vinha sendo perseguida pelo Brasil mesmo antes da Conferência de Amsterdã, uma vez que uma das metas do PCMAM era exatamente aquela de ampliar a capacidade de diagnóstico e tratamento da malária e reduzir a gravidade da doença, e, conseqüentemente, a mortalidade.

De forma semelhante ao que havia sido proposto tanto pelo PCMAM como pela Conferência de Amsterdã, o programa de controle integrado da malária (PCIM) previa: 1) o pronto diagnóstico e o rápido tratamento dos casos da doença; 2) a detecção precoce de epidemias e a aplicação de medidas seletivas de controle; 3 ) o fortaleci- mento da vigilância epidemiológica, a detecção e a prevenção do restabelecimento da transmissão em áreas onde a transmissão da malária fora interrompida; e 4) a reavaliação periódica da situação malárica no país, investindo no conhecimento básico sobre a doença, na capacitação e na organização de serviços descentralizados.

Apesar dos esforços desenvolvidos pelo governo federal e, em muito pequeno número, por governos estaduais e municipais, o PCIM nunca foi plenamente implementado no país. De fato, apenas um de seus componentes foi satisfatoriamente implementado exatamente aquele que previa diagnóstico e tratamento precoce e oportuno. Os demais componentes, como o controle seletivo de vetores, o fortalecimento da vigilância epidemiológica, a intersetorialidade e as ações sobre o meio foram timidamente abordados.

Além disso, o conceito de integralidade das ações raramente foi observado. O programa seguiu um curso de

TABELA 4. Lâminas examinadas e positivas para malária, Brasil, 1995 a 1998

\begin{tabular}{|c|c|c|c|c|c|c|c|c|}
\hline \multirow[b]{2}{*}{ Ano } & \multicolumn{3}{|c|}{ Amostras de sangue } & \multicolumn{5}{|c|}{ Espécies parasitárias } \\
\hline & Examinadas & Positivas & ILPa & Falciparum & $\%{ }^{b}$ & Vivax & $\mathrm{F}+\mathrm{V}^{\mathrm{c}}$ & $M^{d}$ \\
\hline 1995 & 2546708 & 564570 & 22,2 & 199595 & 35 & 360367 & 3843 & 765 \\
\hline 1996 & 2113196 & 444049 & 21,0 & 128418 & 29 & 311208 & 2850 & 1573 \\
\hline 1997 & 1919348 & 405049 & 21,1 & 95439 & 24 & 305491 & 3042 & 1077 \\
\hline 1998 & 2089176 & 471892 & 22,6 & 102718 & 22 & 364435 & 3226 & 1512 \\
\hline
\end{tabular}

Fonte: Gerência Técnica de Malária/Fundação Nacional de Saúde.

a Índice de lâminas positivas por 100 lâminas examinadas.

b Porcentagem da espécie falciparum em relação ao total de espécies identificadas.

c Plasmodium falciparum + Plasmodium vivax.

d Plasmodium malariae. 
ações isoladas, descoordenadas e freqüentemente desprovidas de qualquer análise epidemiológica prévia. Diante de um constante conflito entre dispor de uma proposta técnica bem formulada e a falta de decisão política forte para executar essa proposta de forma integral, ocorreu que, ao longo do tempo, instalou-se um desgaste na confiança e na mística das equipes técnicas, tanto de nível central quanto de nível regional e local.

Somado a isso, observava-se a desmobilização da FUNASA, que passava por um agudo processo de indefinição em relação ao seu futuro e ao seu verdadeiro papel dentro do Sistema Único de Saúde (SUS). Diante desse cenário, como não poderia deixar de ser, a malária voltou a crescer, e isso fez com que a alta direção do Ministério da Saúde e da FUNASA determinassem a elaboração de um novo plano de intensificação das ações de controle. Foi a vez do plano de intensificação das ações de controle da malária nos municípios de alto risco da Amazônia legal $(9,10)$.

\section{Plano de intensificação das ações de controle da malária nas áreas de alto risco da Amazônia legal}

No final de 1996 e início de 1997, a FUNASA elaborou um novo plano de intensificação das ações de controle da malária, de forma semelhante ao que já havia sido tentado em 1993 e 1994 quando, pela primeira vez, foi experimentada a aproximação com os municípios. Também houve um enfoque baseado na estratificação epidemiológica representada por aproximadamente 100 municípios com índice parasitário anual (IPA) maior do que 50 por 1000 habitantes, e em alguns municípios e capitais de estado onde, apesar do IPA menor do que 50 por 1000 habitantes, a situação era bastante grave.

A única diferença entre essa iniciativa e o que propunha o PCIM foi a determinação de fazer convênios com os municípios e, através desse instrumento legal, transferir diretamente aos mesmos os recursos necessários à execução de ações de diagnóstico, trata- mento e controle de vetores. Em 1996 foram celebrados 145 convênios e repassados 16 milhões de reais a estados e municípios enquadrados nas condições estabelecidas pelo plano. Lamentavelmente, a execução de tais convênios não pôde ser acompanhada de perto e, pelo que se soube algum tempo depois, pouquíssimos foram os municípios que realmente cumpriram o que estava proposto nos planos de trabalho.

Muitos municípios utilizaram os recursos de maneira diversa daquela que fora prevista, e outros simplesmente transformaram-se em intermediários para aquisição de materiais e contratação de pessoal que era automaticamente colocado à disposição da FUNASA para que ela passasse a executar as ações. Assim foi contrariado o princípio da descentralização e, com isso, não houve consolidação das estruturas locais que poderiam dar sustentabilidade às atividades de vigilância, prevenção e controle da malária. Diante disso, o impacto esperado com a injeção de recursos novos e o envolvimento dos municípios foi tristemente frustrante, e a malária aumentou ainda mais na região, com uma característica ainda mais grave, que foi o aumento no número de áreas de alto risco.

Em cada uma dessas iniciativas Operação Impacto, estratificação epidemiológica e zonagem, PCMAM, PCIM e plano inicial de intensificação das ações de controle da malária nas áreas de alto risco da Amazônia - é possível destacar o que foi e o que não foi alcançado. Foram alcançadas:

- ampliação da rede de diagnóstico e de tratamento;

- redução da incidência de $P$. falciparum;

- redução do número de internações por malária;

- redução da mortalidade; e

- participação incipiente dos municípios.

Sem dúvida, esses foram ganhos importantíssimos obtidos pelo programa de controle da malária no Brasil. Desde 1989, com o PCMAM, já se ob- servava o esforço realizado para alcançar tais objetivos e, mais do que isso, já se podia verificar, nos anos imediatamente após a sua implementação, o impacto produzido. A oferta de um diagnóstico imediato e de um tratamento oportuno e correto foi significativamente melhorada ao longo do tempo e, nos dias de hoje, já é possível obter diagnósticos em menos de 24 horas em grande parte das áreas trabalhadas.

Além disso, a introdução de drogas mais eficazes e de esquemas de tratamento mais operacionais, como foi o caso da mefloquina para tratamento de $P$. falciparum em áreas de difícil acesso e com populações isoladas, e da aplicação de testes de diagnósticos rápidos, certamente contribuiu significativamente para a obtenção desses resultados.

No entanto, não foram alcançados quatro importantíssimos elementos político-estratégicos:

- intersetorialidade;

- controle seletivo de vetores;

- envolvimento dos estados; e

- sustentabilidade das ações.

A intersetorialidade, por exemplo, é um elemento fundamental para obtenção do sucesso no controle da malária. Assim, o fato de não ter sido alcançada é grave, principalmente quando se sabe que grande parte da produção atual de casos, mais de $60 \%$ na região amazônica, procede de áreas onde estão sendo implementados ou estimulados projetos de assentamento rural de responsabilidade do Instituto Nacional de Colonização e Reforma Agrária (INCRA).

Além dessa situação, houve, também, problemas com invasões em áreas urbanas nas principais capitais de estados da região, como foi o caso de Manaus (capital do Estado do Amazonas), onde o poder público não foi capaz de ordenar as ocupações e nem de dotá-las da infra-estrutura mínima de saneamento e moradia. Ao contrário, facilitou a instalação de aglomerados populacionais totalmente desprotegidos, expondo-os ao risco de adoecer de malária e de outras doenças prevalentes na área. Em ambas as situações não 
houve nenhuma articulação intersetorial para minimizar os possíveis danos provocados por este quadro real e preocupante.

O controle seletivo de vetores também não foi alcançado, ou melhor, perdeu ao longo do tempo a sua importância. Até o final dos anos 1980, havia uma grande preocupação com a aplicação de medidas de controle vetorial, em sua quase totalidade apoiadas na utilização de produtos químicos de ação residual. A partir daí começaram a ser introduzidas, no programa, estratégias de utilização de aplicação espacial com inseticidas químicos através de ultra baixo volume (UBV) e termonebulizações. Estas tiveram o seu apogeu no Estado do Amazonas, onde chegaram a se constituir na principal (e praticamente única) medida de controle.

O controle de vetores, na maioria dos estados da região amazônica, foi perdendo força e qualidade de forma vertiginosa a ponto de chegar nos dias de hoje em níveis que não atingem mais do que $20 \%$ do que realmente se deveria fazer. Saímos de uma situação, na fase da erradicação, de cobertura de $100 \%$ de todas as casas existentes nas áreas maláricas, para uma situação atual crítica, de cobertura mínima de domicílios tratados com inseticida. A normalização e adoção da estratégia do controle seletivo de vetores foram um importante avanço para a racionalidade do uso das medidas. Entretanto, na prática, não se alcançou a sua aplicação devido à falta de equipes suficientes e preparadas para executar essa nova estratégia.

O envolvimento dos governos estaduais, fator decisivo para a sustentabilidade de um programa de controle da malária, também não foi alcançado. Esse foi um erro estratégico cometido pelo governo federal que, de forma semelhante como ocorreu na assistência médica, não valorizou suficientemente o papel dos estados no processo de descentralização do controle da malária e, ao buscar diretamente os municípios, não foi bem sucedido. Convênios com prazo limitado, quando do seu término, não consolidam nenhum compromisso por parte dos municípios, que não conseguem dar prosseguimento, com re- cursos próprios, às atividades até então mantidas com recursos federais transferidos. Isso ocorre em quase todas as situações onde o convênio tem sido o instrumento legal para financiamento de atividades. Normalmente, ao cessarem os efeitos do convênio, cessam também as atividades mantidas por ele. Nesse caso, as atividades sofreram uma redução significativa e, em conseqüência, a incidência da malária cresceu fortemente, chegando a atingir níveis alarmantes (11). De 1998 para 1999, houve um aumento de $26 \%$ no número de casos, de 471892 para 632813 , respectivamente. Além disso, observou-se uma tendência crescente do número de internações por malária, que de 19263 internações em 1998 passou para 21116 em 1999, provavelmente em razão de uma ligeira elevação no número das infecções por $P$. falciparum, que passou de 102719 casos para 117 599, ou seja, um incremento de $19 \%$ em 1999. O que é ainda mais grave, houve um aumento do número de óbitos, de 151 em 1997 para 163 em 1998.

Coincidentemente, no final de 1998 a Organização Pan-Americana da Saúde (OPAS), em consonância com uma iniciativa adotada pela OMS, conhecida como Roll Back Malaria, divulgou uma análise (12) da situação epidemiológica da malária nas Américas e das medidas de controle até então adotadas. O documento analisa, também, o contexto de transformação na maioria dos países, onde políticas de descentralização forçaram a desmobilização dos chamados serviços verticais de controle da malária e deram lugar a uma fase de reestruturação e definição de novos papéis, baseados, principalmente, na organização de serviços locais e permanentes de atenção à saúde das populações. $\mathrm{O}$ objetivo principal desse documento foi chamar a atenção para a necessidade de buscar, imediatamente, mecanismos estratégicos e organizacionais que permitissem manter a malária sob controle, mesmo em um momento de intensa transformação político-institucional. Era necessário "fazer recuar a malária" na região amazônica.

Em seguida, a OPAS anunciou que faria realizar, no período de 18 a 22 de outubro de 1999, na cidade de Lima, no Peru, uma grande reunião internacional para discutir a iniciativa de fazer retroceder a malária nas Américas, com a presença de todos os países amazônicos. Em razão desse anúncio, a representação da OPAS no Brasil tomou a iniciativa de convocar uma reunião no período de 4 e 5 de outubro de 1999, em Brasília, com a participação de renomados pesquisadores e estudiosos da problemática da malária no país, bem como de técnicos da FUNASA, para apresentar e discutir a proposta Roll Back Malaria da OMS e para colaborar com a delegação brasileira que deveria participar da reunião internacional de Lima. Dessa reunião resultou um documento com considerações e recomendações que foi apresentado oficialmente à FUNASA (13).

Como estava previsto, o Brasil compareceu à reunião de Lima, tornandose o centro das atenções, uma vez que o país vinha contribuindo com mais de $40 \%$ de toda a malária nas Américas. Todos os demais países estavam atentos para ver qual seria a proposta do Brasil para fazer recuar a malária a níveis mais confortáveis. Diante do quadro de gravidade, o governo brasileiro resolvera aceitar o desafio, lançando uma proposta extremamente ousada: o plano de intensificação das ações de controle da malária na Amazônia legal (PIACM), comprometendo-se a reduzir em $50 \%$ o número de casos de malária até o final do ano de 2001 em comparação com o ano de 1999 e em $50 \%$ a mortalidade por malária até o final do ano 2002, também em comparação com os dados registrados para o ano de 1999.

\section{PLANO DE INTENSIFICAÇÃO DAS AÇÕES DE CONTROLE DA MALÁRIA NA AMAZÔNIA LEGAL}

O PIACM (11) contém cinco elementos que o diferenciam das iniciativas anteriores:

- forte componente político, ou seja, compromisso político com o con- 
trole da malária assumido pelo presidente da República, governadores de estado e prefeitos de municípios;

- enfoque nas questões do desenvolvimento regional;

- visão do custo social representado pela malária, ou seja, reconhecimento de que a malária não é apenas um problema de saúde, mas sim um problema que afeta o desenvolvimento social;

- importante integração inter e intrasetorial, ou seja, participação de outros setores fora do setor saúde, como por exemplo os ministérios de Meio Ambiente e da Reforma Agrária;

- estratégia estruturadora de serviços;

- sistema de acompanhamento e monitoramento através de avaliações periódicas;

- garantia de financiamento constante e regular pelos governos federal, estaduais e municipais com recursos de seus orçamentos que garantem o financiamento das ações, em valores que variam anualmente dependendo de uma programação pactuada e integrada, elaborada e aprovada pelos três níveis de governo.

O PIACM, assumido, publicamente, pelo presidente da República e pelos ministérios da Saúde, Política Fundiária e Reforma Agrária e Meio Ambiente e Recursos Hídricos, e pelos governadores dos estados da região amazônica (Acre, Amapá, Amazonas, Maranhão, Mato Grosso, Pará, Rondônia, Roraima e Tocantins), tem grandes possibilidades de permitir uma reorganização da luta contra a malária no país. Diferentemente das outras iniciativas, esta tem uma característica muito mais clara, mais vigorosa e transparente de parceira entre os níveis federal, estadual e municipal. Também há uma nítida definição da participação dos outros setores, principalmente do INCRA e do Ministério do Meio Ambiente, o que, certamente, garantirá a intersetorialidade na execução das ações. Não há dúvida de que a visão do custo social da malária foi um fator decisivo para que os governos federal, estaduais e municipais se posicionassem decisivamente a favor do plano.

Observa-se, também, que há uma possibilidade mais concreta, ao contrário daquilo que se constatou nas iniciativas anteriores, de estruturação de serviços. O envolvimento do departamento de ações básicas de saúde do Ministério da Saúde, através dos programas de saúde da família e de agentes comunitários de saúde, será certamente um elemento muito forte para que as ações de prevenção, vigilância e controle da malária sejam, definitivamente, incorporadas à rede de serviços de saúde e deixem de ser responsabilidade isolada do governo federal.

Com a promulgação da Constituição Federal, em 1988, onde ficou definida e assegurada a política de saúde para o país, com a criação do SUS, cujo mais forte pilar é a descentralização, não há mais espaço legal, no Brasil, para sustentar um programa vertical de controle da malária. Dentro do plano há, também, uma importante preocupação com o seguimento da sua implementação e de sua execução. Assim, o plano prevê um sistema de acompanhamento e monitoramento, além de uma avaliação de resultados e de impacto melhor planejada. Nesse sentido, a FUNASA constituiu um comitê técnico assessor com representantes de universidades, da representação da OPAS no Brasil, de entidades como a Sociedade Brasileira de Medicina Tropical (SBMT) e a Associação Brasileira de Pós-Graduação em Saúde Coletiva (ABRASCO) e de Centros de Pesquisa para acompanhar e avaliar o andamento do plano e propor possíveis mudanças de rumo e adoção de novas estratégias de intervenção, caso sejam necessárias. Essa medida não só assegurará uma melhor possibilidade de sucesso do plano, como também produzirá um maior envolvimento das entidades representativas da área de ensino e pesquisa com o serviço, comprometendo-as com as ações do governo para o controle efetivo da malária na região amazônica. Além disso, a iniciativa teve um reforço importantíssimo pela garantia de recursos federais mais constantes e regulares, mediante regulamentação, através da Portaria 1399/99 do Ministério da Saúde e da Portaria 950/99 da FUNASA, do mecanismo de custeio das ações de vigilância epidemiológica e de controle de doenças a serem desenvolvidas por estados e municípios.

\section{Resultados preliminares}

Os dados preliminares disponíveis sobre a incidência da malária na região amazônica brasileira em 2001 revelam um inversão drástica da tendência crescente da doença. Em 1999 foram registrados 630985 casos nos nove estados incluídos no PIACM. Em 2001, foram registrados 383654 casos, uma redução de 39\%, conforme o Sistema de Informação de Malária (SISMAL) do Centro Nacional de Epidemiologia da FUNASA. A redução ocorreu em todos os estados. A menor redução foi verificada no Amapá (15\%), onde o plano só foi implantado no $2^{\circ}$ semestre de 2001. Em relação à incidência por espécies, a redução global de casos por $P$. falciparum foi de $35 \%$ e por $P$. vivax, de $41 \%$.

Nunca na história do controle da malária na Amazônia, desde 1960, houve uma redução tão elevada na transmissão da malária. Para explicar tal redução, a única variável independente reconhecida foi a implantação do PIACM a partir de 2000 (com exceção do Estado do Amazonas, onde já estava em curso, desde 1999, um plano local de aprimoramento do controle da malária com as mesmas características do atual PIACM).

A sustentabilidade desses ganhos ao longo dos próximos anos é ainda uma incógnita. Porém, o fortalecimento dos serviços locais de saúde em toda a região, não somente em abrangência, mas também incorporando as ações de controle de endemias, entre elas a malária, é um fator favorável à manutenção de melhoria do seu controle. A importância política e econômica da malária precisa ser permanentemente reavivada, pois é um fator decisivo para garantir recursos financeiros, materiais e humanos mínimos para as atividades de combate às endemias. 


\section{CONCLUSÕES}

É inegável que grandes esforços têm sido feitos pelos governos na luta contra a malária, seja em nível federal, estadual ou municipal. Também não se pode negar que houve um aporte considerável de recursos na tentativa de obter um resultado satisfatório nessa luta. Entretanto, o que se observa ao longo de todas as iniciativas aqui apresentadas e comentadas é a falta de um esforço mais incisivo para a consolidação de estruturas locais de serviços capazes de resolverem os seus próprios problemas. A malária é uma doença de ocorrência local e focal e, portanto, deve ser abordada com critérios que contemplem o cenário mais localizado possível e que permitam mobilizar recursos locais. No Brasil, as decisões centralizadas, situadas em nível federal, podem ter resolvido, no passado, grande parte do problema quando ainda se dispunha de um serviço bem organizado e estruturado para ser absolutamente vertical, momento em que a cobertura populacional por serviços permanentes de saúde estaduais e municipais era ainda muito baixa e precária. Essa pode ser ainda a realidade em outros países, especialmente de dimensões menores do que o Brasil. No momento, contudo, o Brasil está apostando na construção de um novo modelo, que seja descentralizado, que garanta uma boa qualidade na prestação dos serviços, que assegure uma ação continuada, que perceba as características locais e que tenha muito claramente definidas as responsabilidades de cada um dos partícipes desse modelo. Embora ainda precoces, os resultados apresentados até aqui sugerem um considerável ganho com a iniciativa do governo brasileiro de implementação do PIACM, onde uma forte mobilização política dos três níveis de governo - federal, estadual e municipal — foi decisiva.

\section{REFERÊNCIAS}

1. Fundação Instituto Brasileiro de Geografia e Estatística (IBGE). Anuário Estatístico do Brasil. Rio de Janeiro: IBGE; 1993.

2. Marques AC, Gutierrez HC. Combate à malária no Brasil: evolução, situação atual e perspectivas. Rev Soc Bras Med Trop 1994; 27(Supl III):91-108.

3. Brasil, Superintendência de Campanhas de Saúde Pública (SUCAM), Diretoria Regional do Pará. Relatório sobre a estratificação epidemiológica e sistema operacional por zonagem, 1988. Brasília: Ministério da Saúde; 1988.

4. Marques AC. O controle das grandes endemias em 1984. Rev Bras Malariol Doencas Trop 1985;37:77-92.

5. Passos ADC, Fialho RR. Malária: aspectos epidemiológicos e de controle. Rev Soc Bras Med Trop 1988;31(supl II):93-105.

6. Brasil, Superintendência de Campanhas de Saúde Pública (SUCAM), Diretoria Regional de Rondônia. Manual do guarda medicador.

Operação Impacto Contra a Malária. Brasília: Ministério da Saúde; 1986.

7. Brasil, Superintendência de Campanhas de Saúde Pública (SUCAM). Projeto de Controle da Malária na Bacia Amazônica - PCMAM. Brasília: Ministério da Saúde, 1989.

8. Akavan D. Análise de custo efetividade do Projeto de Controle da Malária na Bacia Amazônica (PCMAM). Brasília: OPAS; 1997.

9. Brasil, Ministério da Saúde, Fundação Nacional de Saúde (FUNASA). Plano de Intensificação das Ações de Controle da Malária nos Municípios de Alto Risco da Amazônica Legal - documento técnico. Brasília: FUNASA; 1996.

10. Brasil, Ministério da Saúde, Fundação Nacional de Saúde (FUNASA). Controle da malária: diretrizes técnicas. $1^{\text {a }}$ ed. Brasília: FUNASA; 1995.

11. Brasil, Fundação Nacional de Saúde (FUNASA). Plano de Intensificação das Ações de
Controle da Malária na Amazônia Legal: documento técnico. Brasília: FUNASA; 2000. Disponível na Internet em http://www. funasa.gov.br/epi/malaria/malaria0.htm. Acessado em 2 de março de 2002.

12. Regional overview of malaria control in the Americas. Roll Back Malaria in the Americas, $\mathrm{PAHO} / \mathrm{HCP} / \mathrm{HCT} / 127 / 98-99$. Washington, DC: PAHO/OMS; 1998.

13. Organização Pan-Americana da Saúde (OPAS). Relatório final da reunião realizada em Brasília - DF, para discutir a iniciativa de Roll Back Malária. Brasília: OPAS; 1999.

Manuscrito recebido em 13 de agosto de 2001. Aceito em versão revisada em 29 de janeiro de 2002. 
ABSTRACT This paper reviews malaria control initiatives in Brazil, from the Malaria Eradication Campaign (Campanha de Erradicação da Malária), which was launched in 1965 and was based on spraying dichlorodiphenyltrichloroethane (DDT) and on administering anti-

Malaria control in Brazil: 1965 to 2001 malarial drugs, to the implementation, in 2000, of the Program for Intensification of Malaria Control in the nine-state Legal Amazon region of Brazil (Plano de Intensificação das Ações de Controle da Malária na Amazônia Legal), which was implemented in response to the World Health Organization's Roll Back Malaria effort. Among the Brazilian initiatives discussed are epidemiological stratification, the Impact Operation (Operação Impacto), the Amazon Basin Malaria Control Project (Projeto de Controle da Malária na Bacia Amazônica), and the Integrated Malaria Control Program (Programa de Controle Integrado da Malária). Although there was progress in the control of malaria before the Intensification Program was launched in 2000, the actions carried out were not sustained. From 1998 to 1999 there was even a 34\% increase in the number of malaria cases in the Brazilian Amazon. The Intensification Program set a goal, in comparison to 1999, of reducing by $50 \%$ the number of malaria cases by the end of 2001 and of cutting by $50 \%$ the mortality due to malaria by the end of 2002. Data for 2001 showed an overall $39 \%$ decrease in the number of malaria cases in the nine Amazonian states of the Intensification Program. The smallest decrease $(15 \%)$ was in the state of Amapá, where the plan was not implemented until the second half of 2001. In terms of incidence by species, there was a 35\% reduction in cases caused by Plasmodium falciparum and a $41 \%$ reduction in cases caused by $P$. vivax. The only independent variable that explains this reduction is the implementation of the Intensification Program. Although preliminary, these results indicate considerable gains. Decisive to this progress has been the strong mobilization of federal, state, and municipal governments.

\section{PAHO Training Program in International Health 2003}

Interested persons are invited to apply for the Training Program in International Health of the Pan American Health Organization (PAHO). The program is intended for professionals from PAHO Member Countries who are no more than 35 years old, have a master's degree in public health or its equivalent, and have at least 2 years of experience in health services, education, or research. The program follows a work-study format, with each participant being incorporated into the work of a technical unit at the PAHO Headquarters office in Washington, D.C., for a period of 11 months.

Applicants must have an excellent command of either Spanish or English and be able to read, write, speak, and understand the other language. Persons interested in being considered for the program must complete an application form and supply a number of other documents substantiating their experience and qualifications.

The program begins each year in late January and ends in mid-December. Participants receive payments that cover their living costs and some other expenses.

The deadline to apply for the 2003 program is 31 July 2002.

Information:

Training Program in International Health

Human Resources Development Program

Division of Health Systems and Services Development

Pan American Health Organization

525 23rd Street, N.W.

Washington, D.C. 20037-2895, United States of America

Telephone: (202) 974-3592

Fax: (202) 974-3612

Web site: http://www.paho.org/English/HSP/HSR/pfsiabout.htm 